\title{
Biofilms in Water Hoses of a Meat Processing Environment Harbor Complex Microbial Communities
}

\author{
Eva M. Voglauer ${ }^{1}$, Benjamin Zwirzitz ${ }^{1 \dagger}$, Sarah Thalguter ${ }^{1}$, Evelyne Selberherr ${ }^{2}$, \\ Martin Wagner ${ }^{1,2}$ and Kathrin Rychli ${ }^{2 *}$
}

'FFoQSI GmbH-Austrian Competence Centre for Feed and Food Quality, Safety and Innovation, Tulln, Austria, ${ }^{2}$ Unit of Food Microbiology, Institute for Food Safety, Food Technology and Veterinary Public Health, University of Veterinary Medicine Vienna, Vienna, Austria

OPEN ACCESS

Edited by:

Faizan Ahmed Sadiq,

Jiangnan University,

China

Reviewed by:

Markus Dieser,

Montana State University,

United States

Xinglian Xu,

Nanjing Agricultural University, China

${ }^{*}$ Correspondence: Kathrin Rychli

kathrin.rychli@vetmeduni.ac.at

tPresent address:

Benjamin Zwirzitz,

University of Natural Resources and

Life Sciences, Institute of Food

Science, Vienna, Austria

Specialty section:

This article was submitted to

Food Microbiology,

a section of the journal

Frontiers in Microbiology

Received: 09 December 2021

Accepted: 20 January 2022

Published: 14 February 2022

Citation:

Voglauer EM, Zwirzitz B, Thalguter S,

Selberherr $E$, Wagner $M$ and

Rychli K (2022) Biofilms in Water

Hoses of a Meat Processing

Environment Harbor Complex

Microbial Communities.

Front. Microbiol. 13:832213.

doi: 10.3389/fmicb.2022.832213
Safe and hygienic water distribution is essential for maintaining product quality and safety. It is known that biofilms alter the appearance and microbial quality of water along the distribution chain. Yet, biofilms in water hoses throughout the food processing environment have not been investigated in detail. Here, microbial communities from water hoses and other environmental sites in contact with water, in addition to the source water itself, were studied in the meat processing environment. Biofilms were present in all water hoses as determined by the presence of bacterial DNA and biofilm matrix components (carbohydrates, extracellular DNA, and proteins). The microbial community of the biofilms was dominated by Proteobacteria, represented mainly by Comamonadaceae and Pseudoxanthomonas. Moreover, genera that are associated with an intracellular lifestyle (e.g., Neochlamydia and Legionella) were present. Overall, the microbial community of biofilms was less diverse than the water microbial community, while those from the different sample sites were distinct from each other. Indeed, only a few phyla were shared between the water hose biofilm and the source water or associated environmental samples. This study provides first insights towards understanding the microbiota of water hose biofilms in the food processing environment.

Keywords: biofilm matrix, contamination source, food spoilage, facultative pathogenic bacteria, intracellular bacteria

\section{INTRODUCTION}

Ensuring water safety is an indispensable aspect of public health. In 2020, two billion people lacked safely managed water; therefore, the access to drinking water is included in the Sustainable Development Goals (SDG6). Even in countries with established water infrastructure, the water reaching the consumer is not guaranteed to be of high quality (United Nations, 2021).

There are various sources of contamination along the water transport chain, including physical (heavy metal particles), chemical (pesticides), and biological contaminants (microorganisms or their toxins; Bhagwat, 2019). Bacterial biofilms in water distribution systems, plumbing systems, and the last meters before water retrieval, e.g., water hoses, have recently been receiving particular attention (Carpentier and Cerf, 1993; Ren et al., 2015; Husband et al., 2016; 
Neu et al., 2019). They are responsible for the deterioration of drinking water quality, in terms of microbial safety and appearance, and the corrosion of pipes. Moreover, they decrease water carrying capacity and thereby lead to increased energy needs (Kumar and Anand, 1998; Wingender and Flemming, 2011; Kip and Van Veen, 2015; Husband et al., 2016). As water system biofilms develop in environments with low nutrient sources and high stresses, such as shear forces, disinfecting agents, and temperature variations, they are also of interest in studying bacterial adaption and resilience.

In various environments, biofilms have been detected in installations for water retrieval. In recent years, studies on biofilms in shower and garden hoses have highlighted the complexity and diversity at the last step in water distribution (Thomas et al., 2014; Soto-Giron et al., 2016; Proctor et al., 2016, 2018; Neu et al., 2019). The major cause for concern for biofilms in water hoses or water distribution systems is that bacteria or clusters thereof can detach, resulting in the contamination of water. Studies have shown that microorganisms from pipe biofilms appear in drinking water (among others Chan et al., 2019; Fish et al., 2020; Wingender and Flemming, 2004). Furthermore, the presence of pathogens in water hose biofilms has been reported, highlighting the potential health threat posed by these biofilms (Thomas et al., 2014; Soto-Giron et al., 2016).

In the food industry, the quality and safety of water are essential for maintaining product quality. Water is used for primary food production, during processing operations, as a food ingredient, and for cleaning and disinfection procedures (Kirby et al., 2003; Bhagwat, 2019).

In large-scale food production, water hoses are used to retrieve water. As in other water-associated environments, biofilms can form, potentially leading to contamination of food products or equipment. Within the food processing environment, two main usages for water hoses exist. Firstly, there are those used for cleaning and disinfection, where the water is typically hot and treated with disinfectants. Secondly, water can flow through hoses attached directly to certain machines. These hoses can be seen as food contact surfaces, as the water passing through them comes directly into contact with the food product itself or the surface on which the food product is processed. To date, these water hoses in the food processing environment have received little attention in regards to microbiological safety and quality investigations. In a recent study, we identified the nozzles of such water hoses to harbor multi-species biofilms (Wagner et al., 2020).

The overall goal of this study was to gain insight into biofilms inside water hoses used in a meat processing environment. Therefore, we characterized biofilms from seven water hoses in a meat processing facility that used unchlorinated water. The bacterial load and the presence of three matrix components (carbohydrates, proteins, and eDNA) were determined. We also examined the microbial community of the hoses via high-throughput 16S rRNA gene sequencing. Additionally, the microbial communities of the source water and various other environmental sites were characterized throughout the facility. Knowledge about the microbial communities of water hoses in the food producing environment can help to prevent and understand contamination events during production.

\section{MATERIALS AND METHODS}

\section{Sampling}

Samples (Figure 1) were taken in July 2020 in an Austrian meat processing facility shortly after the end of production, after regular cleaning and disinfection. Three types of samples were taken: biofilms from water hoses $(\mathrm{H}, n=7)$, water (W, $n=14$ ), and environmental surfaces (E, $n=11)$.

\section{Biofilm Sampling}

Water hoses consisted of multiple layers, the innermost layer consisted of polyvinyl chloride (PVC). Water hoses were removed from the fittings and cut. One meter of each hose was put into a separate bag and transported to the laboratory. Biofilm samples were stored at $4^{\circ} \mathrm{C}$ until further processing within $24 \mathrm{~h}$. Using a sterilized knife, $30 \mathrm{~cm}$ of the hose were cut. The hoses had an inner diameter of $1.2-1.5 \mathrm{~cm}$. The water hoses were sampled according to the scraper-flocked swab method (Maes et al., 2017; Supplementary Figure 1; Supplementary Table 1). A wetted swab was used to hydrate the biofilms before subsequent sampling with scrapers and swabs. The sampling devices were transferred into $10 \mathrm{ml} .25 \mathrm{x}$ Ringer solution (B. Braun Austria $\mathrm{GmbH}$ ). Next, $2 \mathrm{~g}$ of cation exchange resin (CER, described in detail in Wagner et al., 2020) was added to loosen the bacterial cells from the extracellular polymeric substances (EPS). Samples with CER were shaken for $20 \mathrm{~min}$ and centrifuged at $3320 \mathrm{rcf}$ for $10 \mathrm{~min}$. The supernatant (EPS-containing solution) was filtered using a $.22 \mu \mathrm{m}$ filter (Filtropur S0.2 Sarstedt AG\& Co KG). The EPS samples and the cell pellets were frozen at $-20^{\circ} \mathrm{C}$ until further processing.

\section{Water Sampling}

For each water sample, approximately $4 \mathrm{~L}$ of water were sampled in autoclaved wide-mouth bottles (Thermo Scientific ${ }^{\mathrm{TM}}$ Nalgene $^{\mathrm{TM}}$ Wide-Mouth Lab Quality PPCO Bottles). Samples were taken before entering any connecting hose. The samples were transported to the lab at $4^{\circ} \mathrm{C}$ and the water was filtered through $.22 \mu \mathrm{m}$ filters (Millipore Type GS .22 $\mu \mathrm{m}$, Merck Millipore; Supplementary Table 1). The filter papers were stored at $-20^{\circ} \mathrm{C}$ until DNA extraction.

\section{Sampling of Environmental Samples}

Polyurethane sponges (SampleRight ${ }^{\mathrm{TM}}$ Sponge Sampler, World Bio Products) and flocked swabs (COPAN) were used for sampling of environmental sites (Supplementary Table 2). The type of sampling device was chosen upon accessibility of the surface. Hydrated polyurethane sponges were used to sample easy accessible areas. The respective area was horizontally and vertically wiped for several seconds, while using sterile gloves and a sterile size template. The swab was put back into the sterile bag and transported to the lab at $4^{\circ} \mathrm{C}$. There, $10 \mathrm{ml}$ of $.25 \mathrm{x}$ Ringer solution was 


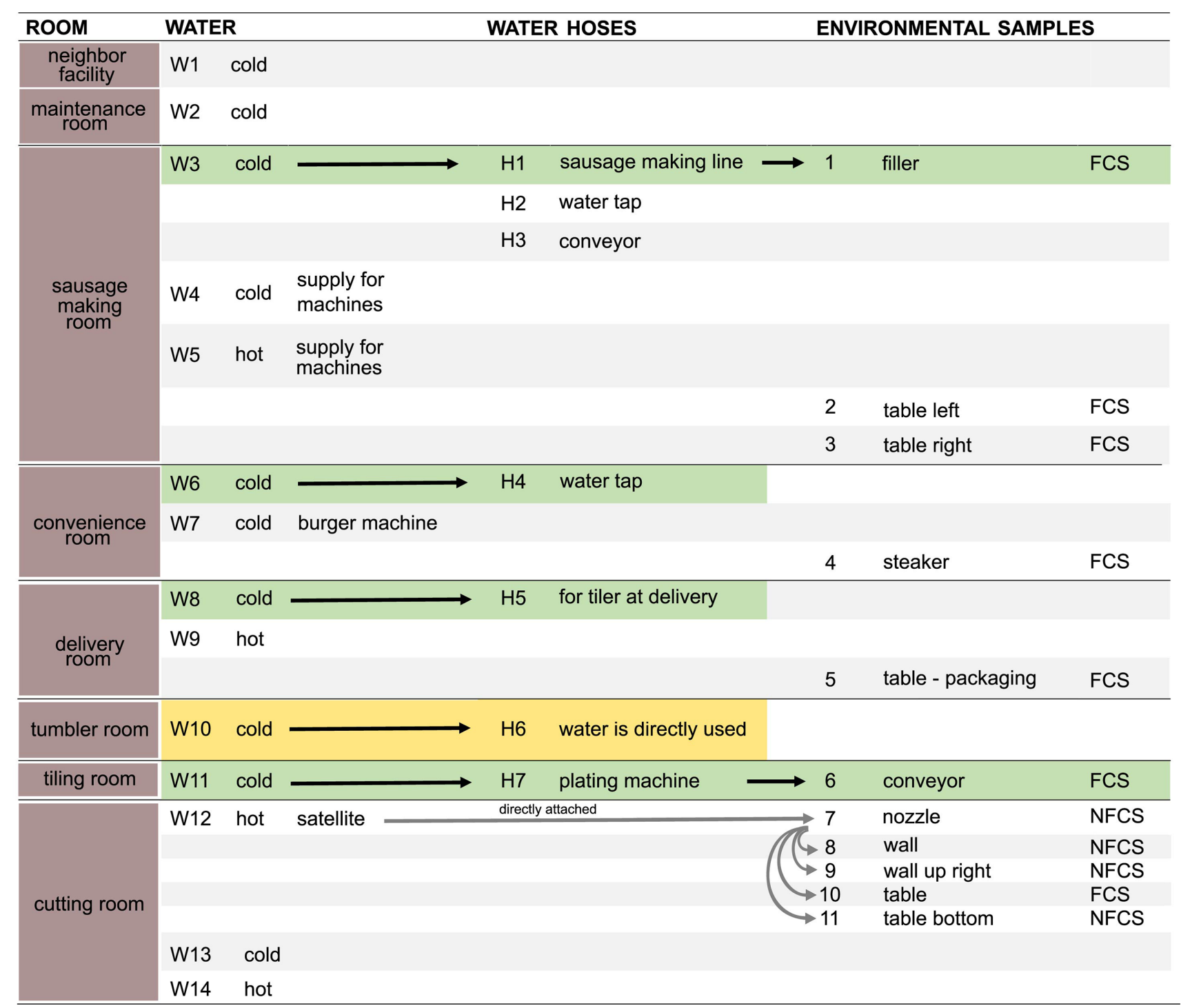

FIGURE 1 | Schematic overview of samples. Water ( $n=14, \mathrm{~W} 1-\mathrm{W} 14)$, water hoses $(n=7, \mathrm{H} 1-\mathrm{H} 7)$, and environmental surfaces $(n=11$, E1-E11) were sampled in different rooms. Arrows indicate a direct transfer of the water through the water hose to the environmental surface. Pairs of directly associated water, hoses, and environmental samples are highlighted in green. Rooms in which water is directly used for food production are highlighted in yellow. Food Contact Surface (FCS); Non-Food Contact Surface (NFCS). Detailed information on environmental samples are found in Supplementary Table 2.

added and the sponge was squeezed extensively to gather the entire liquid. The liquid was then transferred to a $15 \mathrm{ml}$ tube and centrifuged for $10 \mathrm{~min}$ at $3320 \mathrm{rcf}$. The supernatant was removed and the pellet was frozen at $-20^{\circ} \mathrm{C}$ until DNA extraction.

For sites difficult to reach flocked swabs were used. Hereof, multiple devices [in addition to scrapers for larger surfaces areas $\left.\left(50-100 \mathrm{~cm}^{2}\right)\right]$ were used to sample the respective area, by vertically and horizontally wiping for several seconds. The used swabs were added to $10 \mathrm{ml}$ of $.25 \mathrm{x}$ Ringer solution directly after sampling. The samples were stored at $4^{\circ} \mathrm{C}$ and processed within $24 \mathrm{~h}$. In the laboratory, each sample was vortexed for $1 \mathrm{~min}$ and centrifuged for $10 \mathrm{~min}$ at $3220 \mathrm{rcf}$. The swabs and the supernatant were removed and the pellet was frozen at $-20^{\circ} \mathrm{C}$ until DNA extraction.
Negative controls for all sampling types, solutions, and devices were included (Supplementary Table 3).

\section{DNA Extraction}

DNA extraction was carried out using DNeasy PowerSoil Pro Kit (LOT: 166013851) as described previously (Wagner et al., 2020). Samples of each type were extracted at the same time. A negative control (DEPC-treated water) was included for each extraction round. The biofilm pellet was washed using PBS to remove the cation exchange resin (as described by Wagner et al., 2020). The filter papers (of the source water samples) were directly put into the PowerBead Pro tube. For determination of DNA concentration, the Spectrophotometer/Fluorometer DS-11 FX+ (DeNovix) was used. 


\section{Matrix Evaluation}

The presence of EPS components (carbohydrates, proteins, and eDNA) of the water hose biofilm samples was determined. A detailed description on these methods was recently published (Wagner et al., 2020). In short, an aliquot of $1 \mathrm{ml}$ was used for carbohydrate determination using a phenol-sulfuric acid plate assay. The limit of quantification was $79 \mu \mathrm{g} / 10 \mathrm{ml}$ glucose equivalents. Aliquots of $1 \mathrm{ml}$ were used for an overnight protein precipitation using TCA/acetone. The precipitated samples were loaded into an SDS-PAGE (15\%) and subsequently stained using silver-staining. Aliquots of $500 \mu \mathrm{l}$ were precipitated using Na-acetate and ethanol, and quantified using the Spectrophotometer/Fluorometer DS-11 FX+ (DeNovix). For each precipitation and assay controls (i.e., glucose for carbohydrates, BSA for proteins, and salmon sperm DNA for eDNA) were included. Mean values and standard deviations were calculated in $\mathrm{R}$ and visualized using ggplot2 in R (Wickham, 2016).

\section{Enumeration of Bacterial Load Using qPCR}

The total bacterial load, expressed as bacterial cell equivalents (BCE), was determined as previously described (Dixon et al., 2019). A quantitative real-time PCR (qPCR) using the primers $357-\mathrm{F}\left(5^{\prime}\right.$ CCT ACG GGA GGC AGC AG-3') and 518-R(5'-ATT ACC GCG GCT GCT GG-3') to target the 16S rRNA gene (Muyzer et al., 1993) was used (standard curve 1.77 to $6.77 \log \mathrm{BCE}$, primer efficiency 102.8-107.08\%). The reaction mixture consisted of $1 \mathrm{x}$ Brilliant III Ultra-Fast SYBR ${ }^{\circledR}$ Green qPCR Master Mix with Low ROX (Agilent), $250 \mathrm{nM}$ of forward and reverse primer, and $5 \mu \mathrm{l}$ of sample in a $25 \mu \mathrm{l}$ reaction. The samples were analyzed in duplicate using the Stratagene Mx3000P qPCR System with an initial denaturation step at $95^{\circ} \mathrm{C}$ for $3 \mathrm{~min}$. Amplification was carried out in 40 cycles of $3 \mathrm{~s}$ at $95^{\circ} \mathrm{C}$ and $20 \mathrm{~s}$ at $60^{\circ} \mathrm{C}$. A melting curve was performed to identify amplicon specificity $\left(95^{\circ} \mathrm{C}\right.$ for $1 \mathrm{~min}$, $60^{\circ} \mathrm{C}$ for $30 \mathrm{~s}$, and $95^{\circ} \mathrm{C}$ for $30 \mathrm{~s}$ ). In each run, a negative control (DEPC-treated water) was included (Supplementary Table 3). The evaluated copy number of the qPCR control and the respective kit extraction control were subtracted from the respective samples before extrapolation of the total BCE. An average of five $16 \mathrm{~S}$ rRNA gene copies per cell was considered, as estimated using the database for ribosomal RNA operon variation in bacteria and archaea, rrnDB (Větrovský and Baldrian, 2013; Stoddard et al., 2015). Results were visualized using ggplot 2 in $\mathrm{R}$ (Wickham, 2016).

\section{S rRNA Gene Sequencing, Processing, and Analysis}

Amplicon library generation, quality control, and sequencing were performed by the Next-Generation Sequencing Facility at Vienna BioCenter Core Facilities (VBCF), member of the Vienna BioCenter, Austria. The V3V4 region of the 16S rRNA gene was amplified using the primers S-D-Bact-0341-b-S-17 $\mathrm{F}\left(5^{\prime}\right.$-ctctttccetacacgacgctcttccgatct CCTACGGGNGGCWGCAG-3') and S-D-Bact-0785-a-A-21 R(5'-ctggagttcagacgtgtgctcttccgatctGA CTACHVGGGTATCTAATCC-3'; Klindworth et al., 2013). Sequencing was done on an Illumina MiSeq sequencing platform with a $300 \mathrm{bp}$ paired-end read protocol.
Primer and illumina adapter sequences were trimmed from the raw sequence reads with trimmomatic v0.36 (Bolger et al., 2014). The rest of the sequence processing and quality control was performed in $\mathrm{R}$ v3.6.2 using the DADA2 pipeline v1.14 (Callahan et al., 2016; R Core Team, 2020). Briefly, reads with a maximum number of expected errors greater than 2 were removed and the remaining reads were truncated where the average phred quality score dropped below 30 (positions; fwd: 220 and rev: 200). The DADA2 sample-inference algorithm and the following merging of the forward and reverse reads were run with default parameters. Then, chimeras were identified and removed with the "removeBimeraDenovo" command using the consensus method. The remaining reads were annotated to the SILVA SSU database release 138 with a minimum bootstrap threshold of 50 (Quast et al., 2013). Annotated amplicon sequence variants (ASVs) with less than five counts in total were removed from the dataset before continuing analysis. Finally, potential contaminant sequences were identified with the $\mathrm{R}$ package "decontam" using the prevalence method, which compares the prevalence of each sequence in samples to the prevalence in negative controls (Davis et al., 2018). Here, the probability threshold in the "isContaminant" command was set to .5. A total of 309 ASVs were designated as potential contaminants and removed from the dataset (Supplementary Table 4).

Initial data exploration, and basic microbial community analysis, was conducted using the $\mathrm{R}$ package "phyloseq" and a dataset rarefied to the minimum sample size $(5,474$ sequences; McMurdie and Holmes, 2013). Alpha diversity indices were calculated and compared with vegan v2.5-6 (Oksanen et al., 2019) with pairwise comparisons using Wilcoxon rank sum tests and Benjamini-Hochberg adjustment for values of $p$. The dissimilarity in community composition was visualized in ampvis2 v2.6.6 by means of a principal coordinates analysis based on Bray-Curtis dissimilarities as a distance measure (Andersen et al., 2018). Due to not normal distribution of these data and residues, the beta diversity was further assessed by applying a permutational multivariate analysis of variance (PERMANOVA, formerly nonparametric MANOVA) with the adonis function and 5,000 permutations. The relative abundances of individual taxa were calculated and illustrated as barplots (Phyla) or heat maps (Top 50 ASVs) in "phyloseq" and "ampvis2," respectively.

\section{RESULTS}

\section{All Water Hoses Harbored Biofilms}

All seven water hoses $(\mathrm{H} 1-\mathrm{H} 7)$ used in a meat production environment for machines or direct water retrieval had visible biofilms on their inside (Supplementary Figure 1). The bacterial load of all water hose biofilms was above $6.6 \mathrm{log} \mathrm{BCE} / \mathrm{cm}^{2}$ (Figure 2A, minimum $\mathrm{H} 36.6 \pm .1 \log \mathrm{BCE} / \mathrm{cm}^{2}$ ), as determined by quantitative PCR. The highest bacterial cell equivalent count has been detected in the biofilm of water hose $\mathrm{H} 1$ $\left(7.3 \pm .1 \log \mathrm{BCE} / \mathrm{cm}^{2}\right)$. The chemical characterization of the biofilm matrix confirmed the presence of carbohydrates, eDNA, and proteins in all water hoses (Figures $\mathbf{2 B}, \mathbf{C}$, proteins: 
Supplementary Figure 2). The mean matrix carbohydrate load was $3,874 \mathrm{ng}$ glucose equivalents $/ \mathrm{cm}^{2}$ (range from $1,054 \pm 65 \mathrm{ng} /$ $\mathrm{cm}^{2}$ in $\mathrm{H} 2$ to $6,371 \mathrm{ng} / \mathrm{cm}^{2} \pm 195 \mathrm{ng} / \mathrm{cm}^{2}$ in $\left.\mathrm{H} 7\right)$. A mean eDNA load of $64.7 \mathrm{ng} / \mathrm{cm}^{2}$ (range from $15.7 \pm .5 \mathrm{ng} / \mathrm{cm}^{2}$ in $\mathrm{H} 2$ to $122.4 \pm 13.2 \mathrm{ng} / \mathrm{cm}^{2}$ in $\left.\mathrm{H} 7\right)$ was detected. Two of the hoses (H2 and H5) had a notable lower amount of carbohydrates than the other water hoses (Figure 2B, 1,055 \pm 65 and 1,163 $\pm 40 \mathrm{ng} /$ $\mathrm{cm}^{2}$, respectively). Additionally, a lower eDNA amount was observed in $\mathrm{H} 2$ (Figure $2 \mathrm{C}, 15.7 \pm .5 \mathrm{ng} / \mathrm{cm}^{2}$ ). The highest biofilm matrix loads were observed in $\mathrm{H} 7$ with levels of $6,371 \pm 443 \mathrm{ng} /$ $\mathrm{cm}^{2}$ glucose equivalents and $122.4 \pm 13.2 \mathrm{ng} / \mathrm{cm}^{2}$ eDNA.

Within water samples, the mean bacterial cell equivalents were slightly higher in cold water $(n=11,6.4 \pm .7 \log \mathrm{BCE} / \mathrm{l})$ than in hot water $(n=4,6 \pm 1.1 \log \mathrm{BCE} / \mathrm{l}$, Figure 3A). The lowest bacterial load was seen in W12 (4.4 $\pm .04 \log \mathrm{BCE} / \mathrm{l})$, which is part of the cleaning system using hot water. Overall, a low bacterial cell load was detected at the environment sites (Supplementary Table 2). Here, the food contact surfaces $(n=6)$ had a mean bacterial cell equivalent load of $1.64 \pm .59 \log \mathrm{BCE} /$ $\mathrm{cm}^{2}$ and non-food contact surfaces $(n=5)$ of $.5 \pm 1.2 \log \mathrm{BCE} / \mathrm{cm}^{2}$.

\section{Microbial Communities in Water Hose Biofilms}

In total, 4,581,962 16S rRNA gene sequences passed quality control, and 9,336 ASVs were classified, resulting in 101,821 $\pm 76,557$ sequences per sample on average.

The microbial community in water hose biofilms (Figures 4, 5; Supplementary Figures 3 and 4) was dominated by Proteobacteria ranging from $30.2 \%(\mathrm{H} 7)$ to $62.8 \%(\mathrm{H} 5)$. Within this phylum,
A

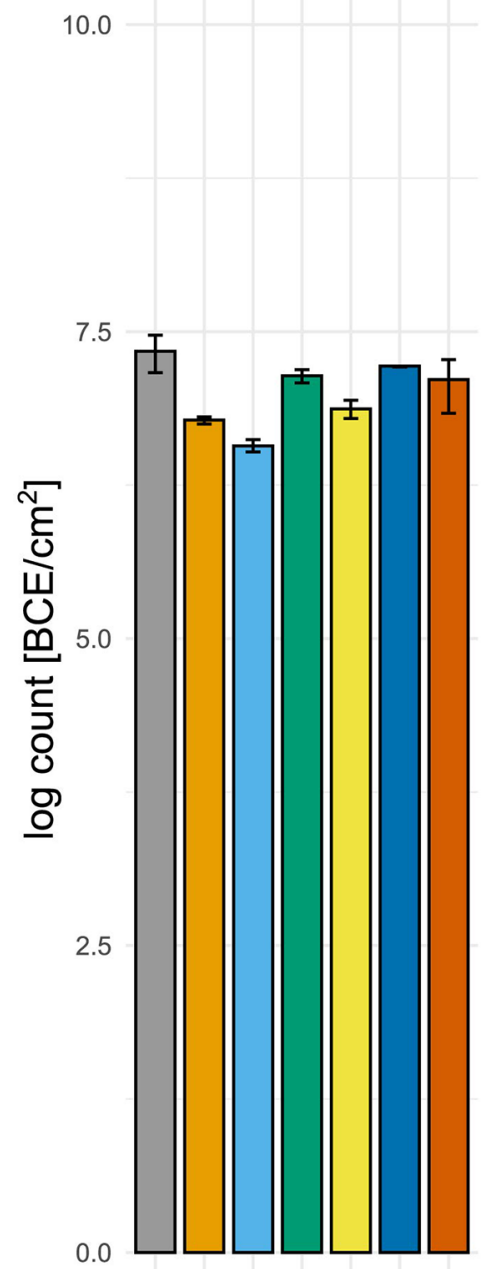

$\mathrm{H} 1 \mathrm{H} 2 \mathrm{H} 3 \mathrm{H} 4 \mathrm{H} 5 \mathrm{H} 6 \mathrm{H} 7$
B

8000

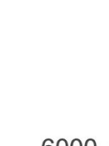

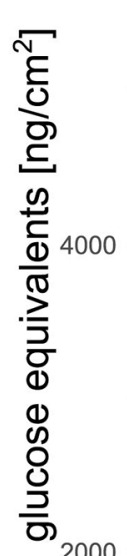

0

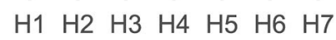

C

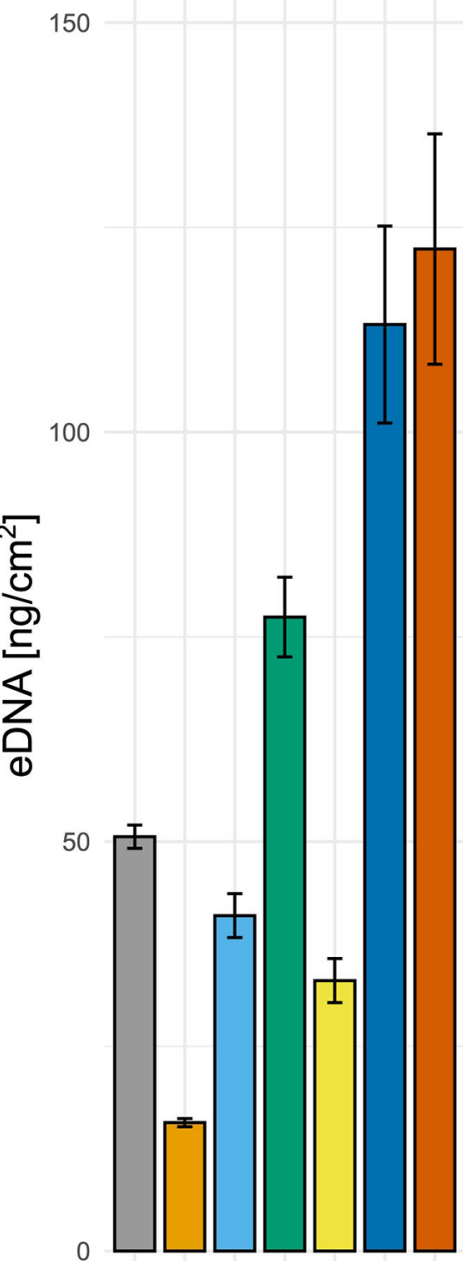

$\begin{array}{llllllll}\mathrm{H} 1 & \mathrm{H} 2 & \mathrm{H} 3 & \mathrm{H} 4 & \mathrm{H} 5 & \mathrm{H} 6 & \mathrm{H} 7\end{array}$

FIGURE 2 | Water hose biofilms. (A) Number of bacterial cell equivalents in biofilms was determined using a qPCR assay targeting the 16S rRNA gene. (B) Matrix polysaccharides were determined using a phenol-sulfuric acid method. (C) The amount of eDNA was spectroscopically measured. Mean values and standard deviation are given of at least two individual measurements. 


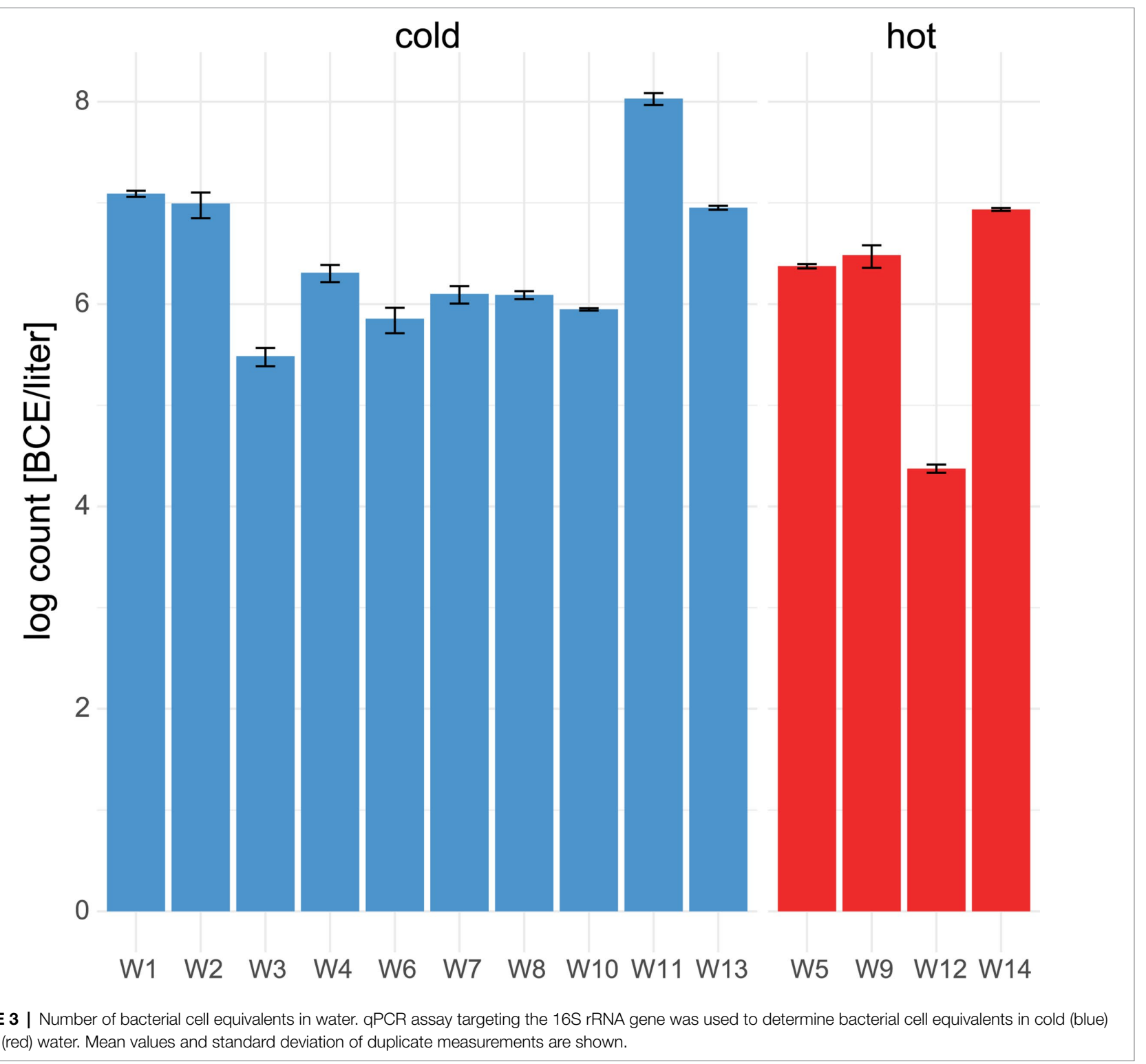

the most abundant genera were related to the Family_ Comamonadaceae (present in five hoses at abundances $>1 \%$ ) and Pseudoxanthomonas (in $\mathrm{H} 2, \mathrm{H} 3, \mathrm{H} 4$, and $\mathrm{H} 6>1 \%$ ). The phylum Verrucomicrobiota accounted for $14.3 \%$ mean relative abundance in water hose biofilms (ranging from $3.7 \%$ in $\mathrm{H} 4$ to $49.4 \%$ in $\mathrm{H} 1$ ) with highest levels of Neochlamydia, which were present in four hoses $\geq 1 \%$, predominantly in H1 (49.4\%) and $\mathrm{H} 3(21.1 \%)$. The third most abundant phylum in water hose biofilms was Planctomycetota [mean relative abundance of $9.5 \%$, ranging from $1.8 \%$ (H1) to $16.2 \%$ (H5)] represented by sequences associated with the genus Gemmata (present in four hoses $>1.7 \%$ abundance), and the family Pirellulaceae (present in 3 hoses $\geq 2.4 \%$ ).

In water hose $\mathrm{H} 6$, the genus Aquabacterium was highly abundant $(25.3 \%)$, which was not the case in the other hoses. Legionella, belonging to the phylum of Proteobacteria, could be detected in all biofilm samples at abundances $\geq .8 \%$.

\section{Microbial Communities in Water Samples}

Also in source water samples, Proteobacteria $(45.7 \%$ in cold and $41 \%$ read abundance in hot water, respectively) presented the most abundant phylum. Within Proteobacteria, the Family_SM2D12 was present in all water samples at relative abundances $>.7 \%$. The next frequent phylum was Patescibacteria $(23.3 \%$ in cold and 29.5\% read abundance in hot water, respectively; Figure 4). Within Patescibacteria, the Order_Saccharimonadales ( $>51 \%$ in cold water, $>.5 \%$ in all hot water samples) and the Order_Candidatus_ Uhrbacteria (present in all water samples $>.3 \%$ ) were the most abundant genera (Supplementary Figure 3).

Sample W1, taken from the neighbor company having the same water supply, and W2, taken from the main service room and representing the first entry site of the water in the meat processing facility, differed from each other. Especially, the genera Order_Saccharimonadales $(10.2 \%$ vs. $1.9 \%$ relative abundance), Family_LWQ (7.9\% vs. $.5 \%$ relative abundance), 


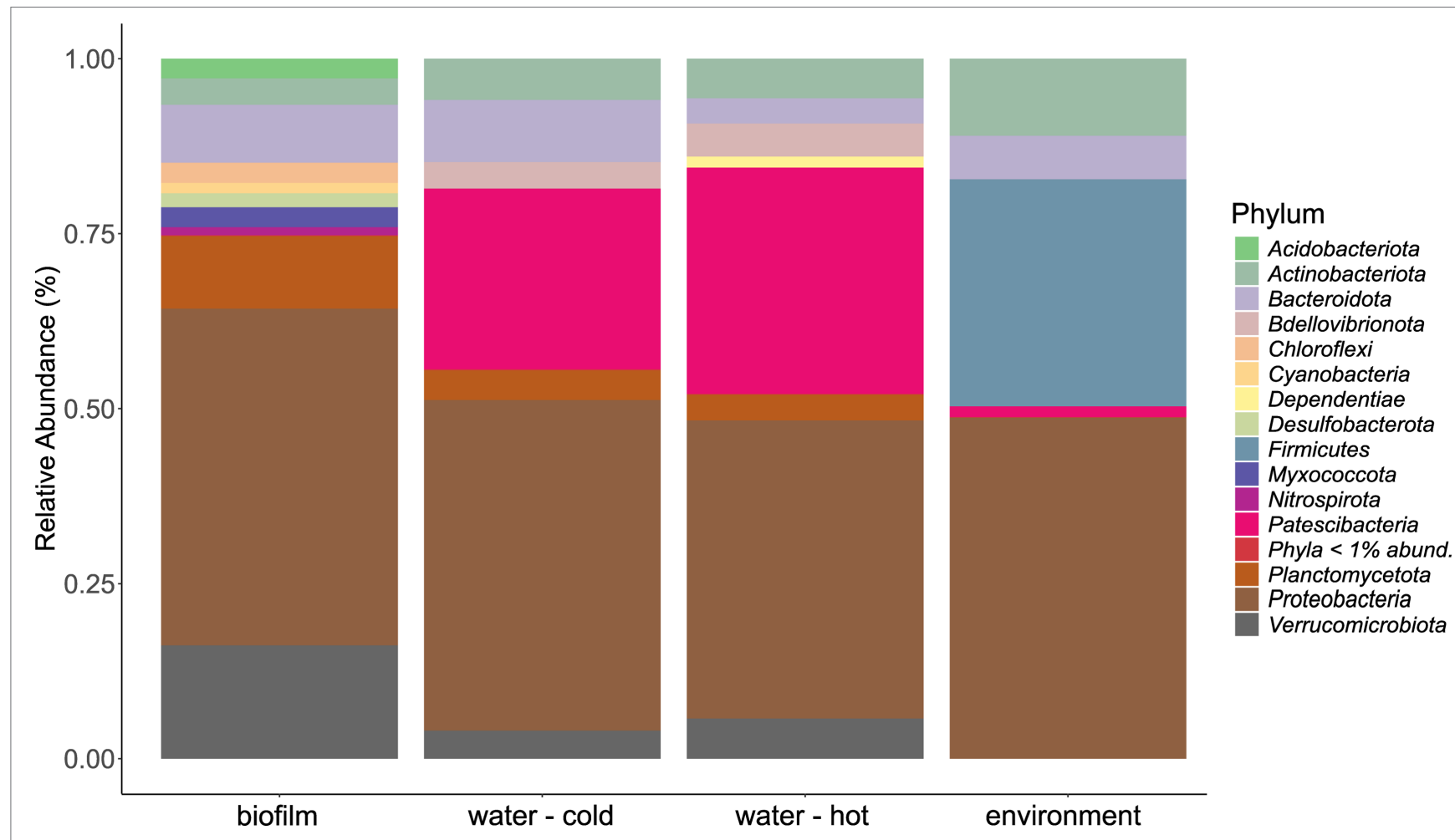

FIGURE 4 | Mean phyla distribution obtained by 16s rRNA gene analysis from microbial communities of water hose biofilms, water, and environmental samples.

Nevskia ( $8.5 \%$ vs. $0 \%$ relative abundance), Novosphingobium (.1\% vs. $17 \%$ relative abundance), and Neochlamydia (.9\% vs. $5 \%$ relative abundance) showed different abundances between these two samples. In water W11, the uncultured phylum KCM-B-112 was predominant $(32.5 \%$ relative abundance); however, this phylum was not observed in high relative abundances in other water samples.

Cold and hot water differed significantly $(p<.05)$ in the abundance of certain genera (Supplementary Figure 6). The presence of Sphingomonas was more prominent in hot water compared to cold water. In cold water several genera, such as Rhodobacter, Legionella, Kocuria, Pseudomonas, Brochothrix etc., were more abundant. Overall, the hot water samples were less diverse than the cold water samples $(6,111$ ASVs in hot vs. 13,913 ASVs in cold water, respectively).

\section{Microbial Communities of Environmental Samples}

Within environmental samples, less phyla with $>1 \%$ read abundance were present $(\mathrm{n}=9)$ than in biofilm samples (Figure 4, $n=11)$. The most prominent phylum was again Proteobacteria (53.7\%), followed by Firmicutes (23.8\%) and Actinobacteriota (12.2\%; Figure 4). The most abundant genera were Pseudomonas (11.7\% mean relative abundance), Photobacterium (6.2\% mean relative abundance), Roseomonas (5\% mean relative abundance), and Acinetobacter (4.4\% mean relative abundance). Single sites were dominated (relative read abundance $>20 \%$ ) by single genera, such as the filler (E1, FCS) by Roseomonas, the steaker
(E4, FCS) and the bottom of the table (E11, NFCS) by Pseudomonas, and the wall of the cutting room (E8, NFCS) by Photobacterium (Supplementary Figure 3 ).

\section{Differences in Community Structure Between Biofilms, Water, and Environmental Samples}

A significant difference in microbial communities was revealed by beta-diversity analysis (Figure 6A, PERMANOVA; betadiversity metric, $\left.R^{2}=.107, d f=1, p=.0002\right)$. The respective sample types clustered, whereas hot and cold water could not be distinguished. The microbial biodiversity was estimated within samples using different alpha diversity indices (observed species, ACE, Shannon, and Simpson index, Figure 6B; Supplementary Table 5). Water samples were overall the most diverse with 862.5 and 959.5 observed ASVs for cold and hot water, respectively. Observed species were significantly lower in biofilms compared to water; however, no significant differences were observed for Shannon and Simpson indices $(p>.05 \%)$. In total, the three different sample types, hose biofilms, water, and environmental sites shared 17 ASVs (ASVs being present in at least seven samples of biofilms and 10 for the other groups with a minimum relative abundance of .01 in each sample type), resulting in a combined relative abundance of $3.3 \%$ (Supplementary Figure 5). Taxonomic classification revealed the genera Brevundimonas, Pseudomonas, Legionella, and Sphingomonas among represented genera (Supplementary Table 6). 


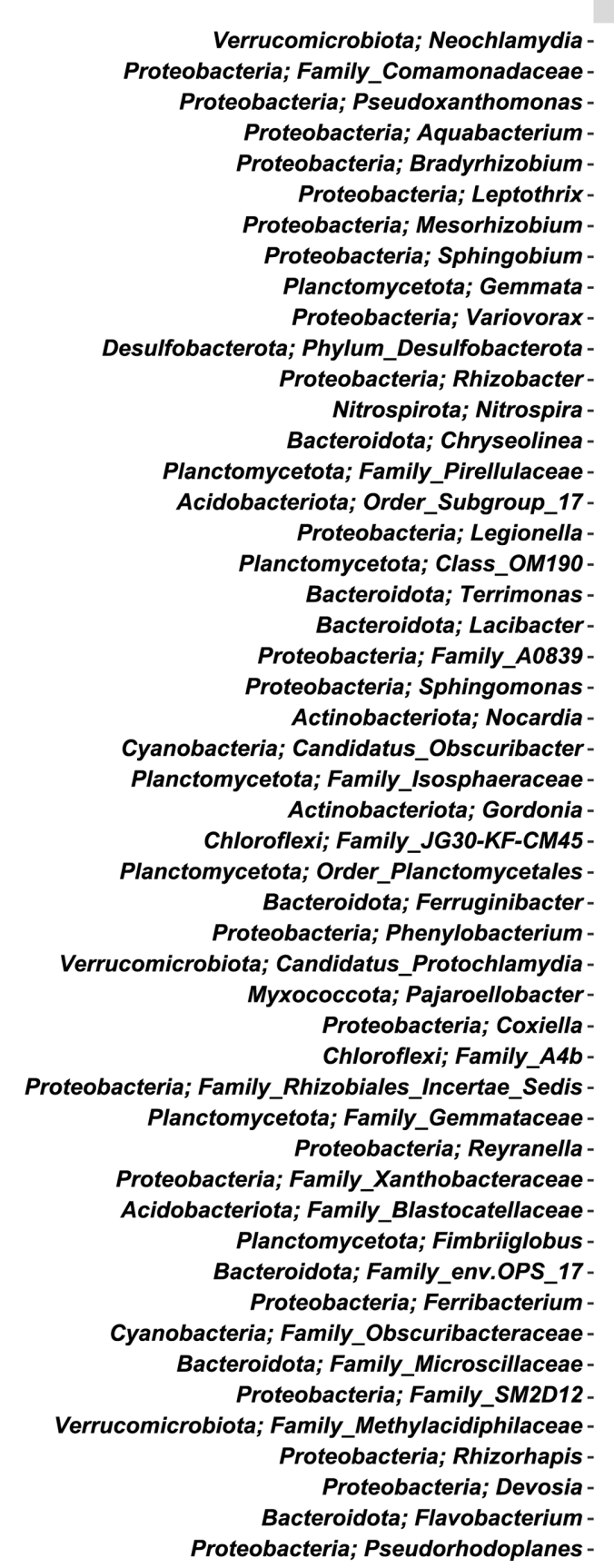

Verrucomicrobiota; Neochlamydia roteobacteria; Pseudoxanthomonas -

Proteobacteria; Aquabacterium Proteobacteria, Bradyrhizobium roteobacteria; Mesorhizobium Proteobacteria; Sphingobium Planctomycetota; Gemmata Proteobacteria, Variovorax Nitrospirota; Nitrospira Planctomycetota; Family_PirellulaceaeAcidobacteriota; Order_Subgroup_17Proteobacteria; Legionella Bacteroidota; Terrimonas Bacteroidota; LacibacterProteobacteria; Family_A0839 Actinobacteriota; Nocardia Cyanobacteria; Candidatus_ObscuribacterPlanctomycetota; Family_IsosphaeraceaeActinobacteriota; Gordonia ( Family JG30-KF-CM45 Bacteroidota; FerruginibacterProteobacteria, Phenylobacterium Myxococcota; PajaroellobacterProteobacteria; Coxiella Chloroflexi; Family_A4bProteobacteria; Family_Rhizobiales_Incertae_Sedis Planctomycetota; Family_GemmataceaeProteobacteria; Reyranella Proteobacteria; Family_XanthobacteraceaePlanctomycetota; Fimbriiglobus Bacteroidota; Family_env.OPS_17Proteobacteria; Ferribacterium cteria; Family_Obscuribacteraceae Proteobacteria; Family_SM2D12. Family_Methylacidiphilaceae Proteobacteria; Devosia Proteobacteria; Pseudorhodoplanes -

\begin{tabular}{|c|c|c|c|c|c|c|c|}
\hline \multicolumn{7}{|c|}{ biofilm } & \\
\hline & 3.9 & 21. & 0.3 & 0.9 & 0 & 1 & \\
\hline 10 & 0.2 & 0 & 13.7 & 1.5 & 1.8 & 2.3 & \\
\hline 0.1 & 17.2 & 5.3 & 4 & 0 & 1.3 & 0 & \\
\hline 0.1 & 0 & 0 & 0 & 0 & 25.3 & 0.1 & \\
\hline 7.9 & 8.2 & 3.3 & 0.1 & 1.3 & 0.3 & 0.4 & \\
\hline 11 & 0.4 & 0 & 7.1 & 0 & 0.5 & 0 & \\
\hline 0 & 0.8 & 11.3 & 0.4 & 0 & 0 & 0 & \\
\hline 0.6 & 1.1 & 0.6 & 3.1 & 3.8 & 2.7 & 0.4 & \\
\hline 1.7 & 2.7 & 3.5 & 1.9 & 0.3 & 0.4 & 0.7 & \\
\hline 0 & 0.1 & 0.2 & 0.1 & 0 & 9.9 & 0 & \\
\hline 0 & 0 & 0.7 & 0.2 & 2.5 & 0 & 6.6 & \\
\hline 0 & 0 & 0 & 0.6 & 2.9 & 0.5 & 5.9 & \\
\hline 0 & 0 & 0 & 0 & 3.3 & 0 & 5.8 & \\
\hline 0 & 0.2 & 1.3 & 4.2 & 0.1 & 1.5 & 1.5 & \\
\hline 0 & 0.1 & 2.9 & 0.4 & 2.9 & 0 & 2.4 & \\
\hline 0 & 0 & 0 & 0 & 1.2 & 0 & 7 & \\
\hline 1.1 & 1.8 & 1 & 0.9 & 1.5 & 0.8 & 1.1 & \\
\hline 0 & 1.4 & 0 & 0.7 & 3.3 & 0.1 & 2.6 & \\
\hline 0 & 0 & 0.4 & 3.3 & 3 & 0.5 & 0.8 & \\
\hline 0.1 & 7.8 & 0 & 0 & 0.1 & 0 & 0 & \\
\hline 0 & 5.3 & 0.2 & 0 & 0.2 & 1.5 & 0 & \\
\hline 0.3 & 0.3 & 3.4 & 1.8 & 0 & 1.1 & 0 & $\%$ Read \\
\hline 0 & 0.2 & 4.9 & 0 & 0 & 0 & 1.8 & \\
\hline 0 & 0 & 0.2 & 3.1 & 0.1 & 3 & 0.6 & 40 \\
\hline 0 & 1.1 & 3.3 & 1.9 & 0.5 & 0.1 & 0 & \\
\hline 0 & 0.2 & 0 & 0 & 0 & 6.3 & 0 & \\
\hline 0 & 2.2 & 3.4 & 0 & 0.2 & 0.3 & 0 & 10 \\
\hline 0 & 0.2 & 1 & 0.1 & 2 & 0 & 2.6 & 5 \\
\hline 0 & 0 & 0.7 & 0.4 & 1 & 3.6 & 0 & 1 \\
\hline 0.1 & 0.1 & 2.6 & 1.9 & 0.2 & 0.9 & 0 & \\
\hline 0 & 2.5 & 0.4 & 0.2 & 0.5 & 0.1 & 1.7 & \\
\hline 0.1 & 0.8 & 1.4 & 0.7 & 0.7 & 0.9 & 0.7 & \\
\hline 0 & 3.9 & 0.7 & 0 & 0.4 & 0 & 0.1 & \\
\hline 0 & 0 & 0 & 2.2 & 1.3 & 0 & 1.3 & \\
\hline 0 & 0.4 & 0 & 0 & 2.2 & 0 & 2.3 & \\
\hline 0 & 0 & 1.7 & 0 & 2 & 0 & 1.1 & \\
\hline 0 & 0 & 1.2 & 0.4 & 1.1 & 0 & 1.8 & \\
\hline 0 & 0.6 & 0.4 & 0 & 2.6 & 0 & 0.8 & \\
\hline 4.2 & 0.1 & 0 & 0 & 0.1 & 0 & 0 & \\
\hline 0.1 & 0 & 0.1 & 0 & 0.6 & 0 & 3.3 & \\
\hline 0.1 & 1.2 & 0 & 0.5 & 0.3 & 0.6 & 1.5 & \\
\hline 0 & 0 & 0 & 3.7 & 0 & 0 & 0.4 & \\
\hline 0 & 0 & 0.1 & 0.3 & 1.3 & 0 & 2.5 & \\
\hline 0 & 0.6 & 0 & 0.4 & 0.5 & 1.7 & 0.9 & \\
\hline 0 & 0.2 & 0 & 3.6 & 0.2 & 0 & 0 & \\
\hline 0 & 0 & 0 & 0 & 0.3 & 3.6 & 0 & \\
\hline 0 & 0.1 & 2.9 & 0 & 0.2 & 0.1 & 0.6 & \\
\hline 0 & 3.2 & 0 & 0 & 0.2 & 0.4 & 0 & \\
\hline 0 & 0.1 & 0 & 0 & 0 & 3.6 & 0 & \\
\hline 0 & 1.6 & 0.4 & 0.1 & 0.7 & 0.9 & 0 & \\
\hline H1 & $\mathrm{H} 2$ & H3 & H4 & H5 & H6 & H7 & \\
\hline
\end{tabular}

FIGURE 5 | Heat map of the top 50 most abundant genera (and their belonging phylum) in biofilm samples. The average ASV counts from replicate 16S rRNA gene libraries for each category are shown. Numbers represent the read abundance in \%.

The hoses shared several genera with the respective water, yet no clear trend between the associated water and the hoses can be observed (Figure 5; Supplementary Figure 3). In the sausage making room, the water hose $\mathrm{H} 1$ and the linked source water W3 shared the first and the second most abundant genera Leptothrix and Family_Comamonadaceae [H1 (11\% and 2.4\%, respectively); W3 (10\% and $1.2 \%$, respectively)]. Yet, the dominating Neochlamydia (49.4\% read abundance) was only observed in the water hose and not in the source water. A limited number of genera were shared between the environmental sites and their linked hoses and water (Supplementary Figure 4). In the sausage making room, Sphingobium was the only phylum that was detected in $\mathrm{H} 1, \mathrm{~W} 3$, and $\mathrm{E} 1 \mathrm{(.6 \%}, 1.4 \%$, and $.8 \%$ read abundance, respectively). Family_Comamonadaceae and 


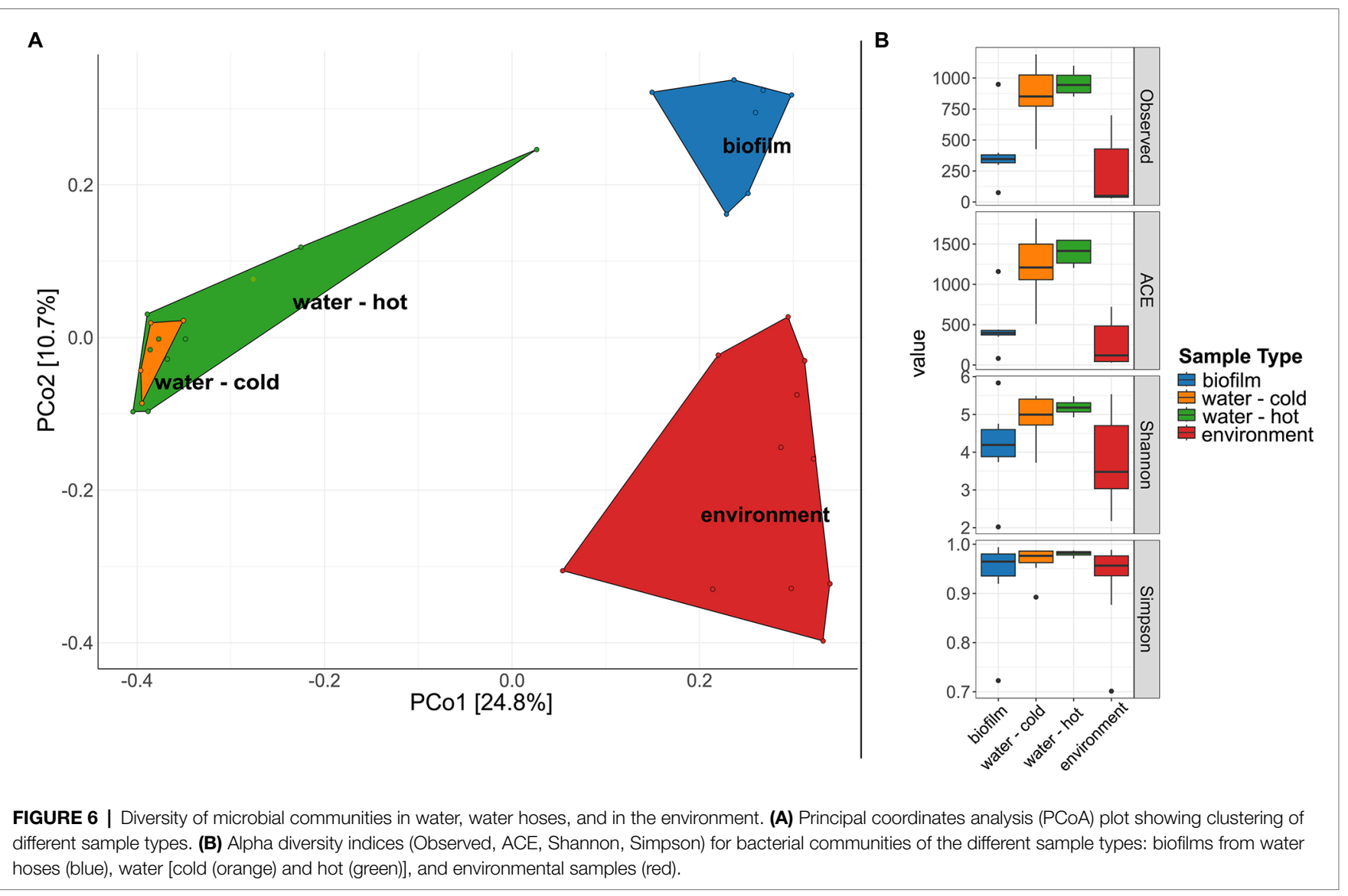

Sphingobium reads were present in most hoses and waters, yet they were hardly detected at environmental sites. Water hose $\mathrm{H} 1$ and its associated environment E1 shared Sphingomonas sequences, in addition to Sphingobium, which was also present in the water,

In the tiling room, $\mathrm{H} 7$ shared the phylum Family_ Microscillaceae with its source water W11 (10.9\% in W11; .9\% in $\mathrm{H7}$ ), yet this phylum could not be detected at the associated environmental site E6. The water hose $\mathrm{H} 7$ and its connected environmental site E6 (the conveyor) shared reads of Family_ Comamonadaceae ( $2.3 \%$ vs. .7\%), Legionella (1.1\% vs. $2.5 \%)$, and Mycobacterium (.3\% vs. $2.5 \%)$ at abundances greater than $.1 \%$ in the hose and associated environmental site.

In the tumbler room, the water (W11) and the associated hose (H6) shared three phyla (abundance $\geq 1 \%$ ). Two phyla were shared between the water and the hose (abundance $\geq 1 \%$ ) in the convenience room (W6-H4) and the delivery room (W8-H5).

\section{DISCUSSION}

The presence of biofilms in water-associated equipment leads to several consequences, such as the deterioration of drinking water quality, the possible transmission of bacteria, corrosion of pipes, and a decrease of water carrying capacity. Studies investigating water hose biofilms are rare and limited to specific environments. Biofilm formation in water hoses has been documented in the hospital setting (Soto-Giron et al., 2016), garden hoses (Thomas et al., 2014), and domestic shower hoses (Moat et al., 2016; Proctor et al., 2016, 2018; Neu et al., 2019). Safe water is indispensable for public health and water quality has a huge impact on food quality and security (Bhagwat, 2019). Water is known to be colonized by bacteria from biofilms on contacting materials, such as pipes (Chan et al., 2019) and drinking water distribution systems (Wingender and Flemming, 2004). This is a crucial concern in food production, as water is used in various processing steps. Within the food processing environment, this is the first study that focuses on the characterization of biofilms in water hoses. We demonstrated that different water hoses, used in the meat processing context for many years, harbored biofilms with a complex and unique microbial community. Biofilms visible to the naked eye could be observed in all water hoses, comparable to other studies dealing with materials in contact with water (Thomas et al., 2014; Neu et al., 2018, 2019; Proctor et al., 2018). The presence of matrix components (carbohydrate, proteins, and extracellular DNA) in the biofilm could be confirmed. These analyses were included to confirm the presence of biofilms, as they are defined to be composed of both microorganisms and matrix. Moreover, these analyses showed that the biofilm matrix of water hose biofilms harbor different amounts of carbohydrates, proteins, and extracellular DNA, which speaks to the complexity of these biofilms. The bacterial load of these water hose biofilms 
was comparable to those identified in studies focusing on biofilms in shower hoses and drinking water distribution network biofilms (Wingender and Flemming, 2004; Proctor et al., 2018). Proteobacteria were predominantly present in all water hose biofilms, its source water and associated environments, which is in line with previous studies focusing on biofilms from water distribution and plumbing systems, as well as water contacting materials (Hong et al., 2010; Pinto et al., 2012; Ren et al., 2015; Moat et al., 2016; Proctor et al., 2016, 2018; Neu et al., 2018; de Sotto et al., 2020).

The microbial community of the water hose biofilms in the meat processing environment was diverse and differed between hoses collected in the same room. In all rooms, opportunistic pathogen containing genera, such as Neochlamydia, Legionella, or Pseudomonas, have been detected in different abundances. Neochlamydia, an obligate intracellular symbiont of amoebae, was highly present in the sausage making room, especially in water hose $\mathrm{H} 1$ and water hose $\mathrm{H} 3$. In the other rooms, this genus showed low abundance. In the tiling room, Neochlamydia was present in both the water (W11) and the linked water hose (H7), which could indicate a possible transmission. Another facultative intracellular pathogenic genus detected in all water hoses and the source water was Legionella, whose presence has been described in domestic shower hoses (Proctor et al., 2016, 2018), garden hoses (Thomas et al., 2014), and cooling towers (Tsao et al., 2019). The presence of Legionella has previously been reported to correlate with total cell counts (Proctor et al., 2018). Therefore, thick biofilms might act as a means of proliferation for Legionella (van der Kooij et al., 2017). The presence of facultative and obligate intracellular bacteria in these water hoses indicate the presence of protozoa in the water hose biofilms, as reported in a previous study on garden water hoses (Thomas et al., 2014). The role of amoebae in water-associated biofilms has already been investigated (Thomas et al., 2010, 2014; Morsy et al., 2016; Proctor et al., 2018; Taravaud et al., 2018; Shaheen et al., 2019), yet their definite role in the transfer of pathogenic bacteria in water-associated environments remains to be elucidated.

The detection of several other genera is in line with publications investigating different water-associated biofilms. Pseudoxanthomonas was also detected in hospital shower hoses (Soto-Giron et al., 2016), Sphingobium in drinking water distribution system (Fish et al., 2020) and hospital shower hoses (Soto-Giron et al., 2016), and Bradyrhizobium in hospital shower hoses (Soto-Giron et al., 2016), in domestic shower hoses (Moat et al., 2016; Proctor et al., 2018), in laboratory studies (Neu et al., 2019; Fish et al., 2020), and bath toys (Neu et al., 2018). Sphingomonas, which was present in three water hoses of the meat processing environment at relative abundances $>1 \%$, was also described to be present in hospital shower hoses (Soto-Giron et al., 2016), in domestic shower hoses (Moat et al., 2016; Proctor et al., 2018), and laboratory studies (Neu et al., 2019), as well as biofilms on plastic bath toys (Neu et al., 2018).

In a previous study, focusing on the detection of biofilms in the meat processing environment, we cultivated meatspoilage bacteria, such as Microbacterium, Stenotrophomonas, Brochothrix, and Psychrobacter, from biofilms on water hose nozzles (Wagner et al., 2020). In the water hose biofilms of the present study, we also detected Pseudomonas, Microbacterium, and Psychrobacter. Pseudomonas and Microbacterium in two water hoses, and Pseudomonas in the supplying water (W3 and W11, respectively). However, other genera linked to meat spoilage, such as Lactobacillus, Acetobacter, Kocuria, Lactococcus, Stenotrophomonas, and Carnobacterium (Maes et al., 2019), have not been detected in water hose biofilms. Within a laboratory-based study, we could demonstrate that isolates from the genera Microbacterium and Stenotrophomonas were able to form mono-species biofilms on stainless-steel slides under conditions mimicking the food processing environment (Wagner et al., 2021). Additionally, we analyzed the microbial composition of water, where five samples were directly associated with the water hoses. The $16 \mathrm{~S}$ rRNA gene load from the water was comparable with those identified by Waak et al. in non-treated water (Waak et al., 2019). The microbial community of the water had a higher species richness than water hoses, which is consistent with other studies (Proctor et al., 2018). Prominent taxa within the water included the Order Saccharimonadales, the Order_Candidatus_Uhrbacteria, and $K C M-B-112$. The fact that representatives of these groups have not been cultivated so far highlights the need of further efforts to cultivate organisms from water distribution systems. The hoses, which had been installed for several years, and their associated water shared only a few phyla (e.g., Leptothrix, Family_Comamonadaceae and Sphingobium). The dominant taxa of the water and the respective water hose biofilm were different. This was also observed in other studies (Waak et al., 2019).

We further observed that the hot water samples were less diverse than the cold water samples, which is in line with a recent study (Proctor et al., 2018). Water poses a risk for food contamination when harboring spoilage bacteria. Previously, the presence of coliforms in wash water for milking equipment was reported to be a risk factor for contamination (Perkins et al., 2009). In this study, ASVs linked to meat-spoilage organisms, such as Pseudomonas (ASV101, ASV912), Brochothrix (ASV37), and Kocuria (ASV68), have been more frequently found in cold than in hot water, indicating the importance of water temperature. Furthermore, Legionella and Mycobacterium have been identified in the water. Even though these bacteria might not pose a problem in terms of food contamination, they might pose a safety issue for workers that inhale the aerosols produced during cleaning and disinfection (Wingender and Flemming, 2004, 2011). These are important findings for the management of water safety in the food industry for both the workers and consumers health.

Despite the water showing high levels of bacterial cell equivalents, the water itself is not the only source for microorganisms in water hose biofilms. Aerosols are capable of transferring microorganisms to water hoses. A study using a GFP-tagged Pseudomonas putida strain showed that this motile bacterium was able to colonize the inside, and the outside of a water hose, after being transferred to the hose by aerosols resulting from high pressure water cleaning 
(Gagnière et al., 2006). This observation together with the evaluation of water hoses as potential contamination sources supports the notion that intervention strategies to prevent colonization of water hoses have to be taken. For example, hindering of contamination by aerosols could be done by storing the exposed openings in disinfecting solutions (Gagnière et al., 2006). The colonization of hoses by microorganisms derived from the water source could be facilitated by different approaches, such as (i) filtering of the water before it enters the hose, (ii) using materials that hinder biofilm formation in hoses, and (iii) the frequent replacement of water hoses.

Additionally, we investigated environmental samples, which showed a low microbial load. Few phyla were shared between the hoses and the associated environmental sites. Pseudomonas, Photobacterium, Acinetobacter, and Roseomonas were more abundant at the environmental sites compared to the biofilm or water samples. With the exception of the Roseomonas, these have already been described as being most abundant in the environment of another meat processing facility (Zwirzitz et al., 2021), indicating that these microbes might have adapted to cleaning and disinfection strategies, and other characteristics of the food processing environment (e.g., cool temperatures). Nevertheless, the observation of 16S rRNA gene sequences of these genera does not allow us to say anything about whether these microbes, are viable as no cultivation-based approaches were applied in this study.

\section{CONCLUSION}

This study demonstrates the complexity of biofilms in water hoses in a meat processing environment. We showed that different water hoses linked to meat processing equipment harbored biofilms with unique microbial communities and different matrix amounts. This study only represents a first snapshot on this issue. Further studies are needed to understand the role of water and water hoses as contamination source in the food producing environment and the role of certain environmental characteristics, such as nutrient sources, materials (e.g., Proctor et al., 2016), temperature, and time, on the formation of biofilms in water hoses.

\section{REFERENCES}

Andersen, K.S., Kirkegaard, R.H., Karst, S.M., and Albertsen, M. (2018). ampvis2: an $\mathrm{R}$ package to analyse and visualise $16 \mathrm{~S}$ rRNA amplicon data. bioRxiv. doi:10.1101/299537.

Bhagwat, V. R. (2019). Safety of water used in food production. Food Saf. Hum. Heal. 2019, 219-247. doi: 10.1016/B978-0-12-816333-7.00009-6

Bolger, A. M., Lohse, M., and Usadel, B. (2014). Trimmomatic: a flexible trimmer for Illumina sequence data. Bioinformatics 30, 2114-2120. doi: 10.1093/ bioinformatics/btu170

Callahan, B. J., McMurdie, P. J., Rosen, M. J., Han, A. W., Johnson, A. J. A., and Holmes, S. P. (2016). DADA2: high-resolution sample inference from Illumina amplicon data. Nat. Methods 13, 581-583. doi: 10.1038/nmeth.3869

Carpentier, B., and Cerf, O. (1993). Biofilms and their consequences, with particular reference to hygiene in the food industry. J. Appl. Bacteriol. 75, 499-511. doi: 10.1111/j.1365-2672.1993.tb01587.x

\section{DATA AVAILABILITY STATEMENT}

The datasets presented in this study can be found in online repositories. The names of the repository/repositories and accession number(s) can be found at: https://www.ebi.ac.uk/ena, PRJEB49015.

\section{AUTHOR CONTRIBUTIONS}

EV, KR, ES, and MW conceived and designed the study. Sampling was performed by EV and KR. EV and ST performed the sample processing. BZ developed bioinformatics pipelines. $\mathrm{BZ}, \mathrm{EV}$, and ST performed data analysis. $\mathrm{BZ}, \mathrm{EV}$, and $\mathrm{KR}$ wrote the manuscript. All authors contributed to the article and approved the submitted version.

\section{FUNDING}

This work was created within a research project of the Austrian Competence Centre for Feed and Food Quality, Safety and Innovation (FFoQSI). The COMET-K1 competence center FFoQSI is funded by the Austrian federal ministries BMK, BMDW, and the Austrian provinces Lower Austria, Upper Austria, and Vienna within the scope of COMET-Competence Centers for Excellent Technologies. The program COMET is handled by the Austrian Research Promotion Agency FFG.

\section{ACKNOWLEDGMENTS}

This research was supported using resources of the VetCore Facility (Genomics) of the University of Veterinary Medicine Vienna. We thank the food processing company for its interest and constructive cooperation. Furthermore, we thank Cameron Strachan for proofreading the manuscript.

\section{SUPPLEMENTARY MATERIAL}

The Supplementary Material for this article can be found online at: https://www.frontiersin.org/articles/10.3389/fmicb.2022. 832213/full\#supplementary-material

Chan, S., Pullerits, K., Keucken, A., Persson, K. M., Paul, C. J., and Rådström, P. (2019). Bacterial release from pipe biofilm in a full-scale drinking water distribution system. NPJ Biofilms Microbiomes 5, 3-10. doi: 10.1038/s41522019-0082-9

Davis, N. M., Proctor, D. M., Holmes, S. P., Relman, D. A., and Callahan, B. J. (2018). Simple statistical identification and removal of contaminant sequences in marker-gene and metagenomics data. Microbiome 6:226. doi: 10.1186/ s40168-018-0605-2

de Sotto, R., Tang, R., and Bae, S. (2020). Biofilms in premise plumbing systems as a double-edged sword: microbial community composition and functional profiling of biofilms in a tropical region. J. Water Health 18, 172-185. doi: 10.2166/wh.2020.182

Dixon, E., Rabanser, I., Dzieciol, M., Zwirzitz, B., Wagner, M., Mann, E., et al. (2019). Reduction potential of steam vacuum and high-pressure water treatment on microbes during beef meat processing. Food Control 106:106728. doi: $10.1016 /$ j.foodcont.2019.106728 
Fish, K. E., Reeves-McLaren, N., Husband, S., and Boxall, J. (2020). Unchartered waters: the unintended impacts of residual chlorine on water quality and biofilms. NPJ Biofilms Microbiomes 6, 34. doi: 10.1038/s41522-020-00144-w

Gagnière, S., Auvray, F., and Carpentier, B. (2006). Spread of a green fluorescent protein-tagged pseudomonas putida in a water pipe following airbone contamination. J. Food Prot. 69, 2692-2696. doi: 10.4315/0362028X-69.11.2692

Hong, P.-Y., Hwang, C., Ling, F., Andersen, G. L., LeChevallier, M. W., and Liu, W.-T. (2010). Pyrosequencing analysis of bacterial biofilm communities in water meters of a drinking water distribution system. Appl. Environ. Microbiol. 76, 5631-5635. doi: 10.1128/AEM.00281-10

Husband, S., Fish, K. E., Douterelo, I., and Boxall, J. (2016). Linking discolouration modelling and biofilm behaviour within drinking water distribution systems. Water Sci. Technol. Water Supply 16, 942-950. doi: $10.2166 /$ ws. 2016.045

Kip, N., and Van Veen, J. A. (2015). The dual role of microbes in corrosion. ISME J. 9, 542-551. doi: 10.1038/ismej.2014.169

Kirby, R. M., Bartram, J., and Carr, R. (2003). Water in food production and processing: quantity and quality concerns. Food Control 14, 283-299. doi: 10.1016/S0956-7135(02)00090-7

Klindworth, A., Pruesse, E., Schweer, T., Peplies, J., Quast, C., Horn, M., et al. (2013). Evaluation of general 16S ribosomal RNA gene PCR primers for classical and next-generation sequencing-based diversity studies. Nucleic Acids Res. 41, e1-e1. doi: 10.1093/nar/gks808

Kumar, C. G., and Anand, S. K. (1998). Significance of microbial biofilms in food industry: a review. Int. J. Food Microbiol. 42, 9-27. doi: 10.1016/ S0168-1605(98)00060-9

Maes, S., Heyndrickx, M., Vackier, T., Steenackers, H., Verplaetse, A., and De Reu, K. (2019). Identification and spoilage potential of the remaining dominant microbiota on food contact surfaces after cleaning and disinfection in different food industries. J. Food Prot. 82, 262-275. doi: 10.4315/0362028X.JFP-18-226

Maes, S., Huu, S. N., Heyndrickx, M., Weyenberg, S.Van, Steenackers, H., Verplaetse, A., et al. (2017). Evaluation of two surface sampling methods for microbiological and chemical analyses to assess the presence of biofilms in food companies. J. Food Prot. 80, 2022-2028. doi:10.4315/0362-028X.JFP-17-210.

McMurdie, P. J., and Holmes, S. (2013). phyloseq: an R package for reproducible interactive analysis and graphics of microbiome census data. PLoS One 8:e61217. doi: 10.1371/journal.pone.0061217

Moat, J., Rizoulis, A., Fox, G., and Upton, M. (2016). Domestic shower hose biofilms contain fungal species capable of causing opportunistic infection. J. Water Health 14, 727-737. doi: 10.2166/wh.2016.297

Morsy, G. H., Al-Herrawy, A. Z., Elsenousy, W. M., and Marouf, M. A. (2016). Prevalence of free-living amoebae in tap water and biofilm, Egypt. Res. J. Pharm. Biol. Chem. Sci. 7, 752-759.

Muyzer, G., De Waal, E. C., and Uitterlinden, A. G. (1993). Profiling of complex microbial populations by denaturing gradient gel electrophoresis analysis of polymerase chain reaction-amplified genes coding for 16S rRNA. Appl. Environ. Microbiol. 59, 695-700. doi: 10.1128/aem.59.3.695-700.1993

Neu, L., Bänziger, C., Proctor, C. R., Zhang, Y., Liu, W. T., and Hammes, F. (2018). Ugly ducklings - The dark side of plastic materials in contact with potable water. NPJ Biofilms Microbiomes 4, 7. doi: 10.1038/s41522-018-0050-9

Neu, L., Proctor, C. R., Walser, J. C., and Hammes, F. (2019). Small-scale heterogeneity in drinking water biofilms. Front. Microbiol. 10:2446. doi: $10.3389 /$ fmicb.2019.02446

Oksanen, J., Blanchet, F. G., Friendly, M., Roeland Kindt, P., Legendre, D. M., Minchin, P. R., et al. (2019). vegan: Community Ecology Package. Available at: https://cran.r-project.org/package=vegan (Accessed August 10, 2021).

Perkins, N. R., Kelton, D. F., Hand, K. J., MacNaughton, G., Berke, O., and Leslie, K. E. (2009). An analysis of the relationship between bulk tank milk quality and wash water quality on dairy farms in Ontario, Canada. J. Dairy Sci. 92, 3714-3722. doi: 10.3168/jds.2009-2030

Pinto, A. J., Xi, C., and Raskin, L. (2012). Bacterial community structure in the drinking water microbiome is governed by filtration processes. Environ. Sci. Technol. 46, 8851-8859. doi: 10.1021/es302042t

Proctor, C. R., Gächter, M., Kötzsch, S., Rölli, F., Sigrist, R., Walser, J. C., et al. (2016). Biofilms in shower hoses-choice of pipe material influences bacterial growth and communities. Environ. Sci. Water Res. Technol. 2, 670-682. doi: 10.1039/c6ew00016a
Proctor, C. R., Reimann, M., Vriens, B., and Hammes, F. (2018). Biofilms in shower hoses. Water Res. 131, 274-286. doi: 10.1016/j.watres.2017.12.027

Quast, C., Pruesse, E., Yilmaz, P., Gerken, J., Schweer, T., Yarza, P., et al. (2013). The SILVA ribosomal RNA gene database project: improved data processing and web-based tools. Nucleic Acids Res. 41, D590-D596. doi: $10.1093 /$ nar/gks1219

R Core Team (2020). R: A language and environment for statistical computing. Available at: https://www.r-project.org/ (Accessed September14, 2021).

Ren, H., Wang, W., Liu, Y., Liu, S., Lou, L., Cheng, D., et al. (2015). Pyrosequencing analysis of bacterial communities in biofilms from different pipe materials in a city drinking water distribution system of East China. Appl. Microbiol. Biotechnol. 99, 10713-10724. doi: 10.1007/s00253-015-6885-6

Shaheen, M., Scott, C., and Ashbolt, N. J. (2019). Long-term persistence of infectious legionella with free-living amoebae in drinking water biofilms. Int. J. Hyg. Environ. Health 222, 678-686. doi: 10.1016/j.ijheh. 2019.04.007

Soto-Giron, M. J., Rodriguez-R, L. M., Luo, C., Elk, M., Ryu, H., Hoelle, J., et al. (2016). Biofilms on hospital shower hoses: characterization and implications for nosocomial infections. Appl. Environ. Microbiol. 82, 2872-2883. doi: 10.1128/AEM.03529-15

Stoddard, S. F., Smith, B. J., Hein, R., Roller, B. R. K., and Schmidt, T. M. (2015). rrnDB : improved tools for interpreting rRNA gene abundance in bacteria and archaea and a new foundation for future development. Nucleic Acids Res. 43, D593-D598. doi: 10.1093/nar/gku1201

Taravaud, A., Ali, M., Lafosse, B., Nicolas, V., Féliers, C., Thibert, S., et al. (2018). Enrichment of free-living amoebae in biofilms developed at upper water levels in drinking water storage towers: An inter- and intra-seasonal study. Sci. Total Environ. 633, 157-166. doi: 10.1016/j.scitotenv.2018.03.178

Thomas, V., McDonnell, G., Denyer, S. P., and Maillard, J. Y. (2010). Free-living amoebae and their intracellular pathogenic microorganisms: risks for water quality. FEMS Microbiol. Rev. 34, 231-259. doi: 10.1111/j.1574-6976.2009.00190.x

Thomas, J. M., Thomas, T., Stuetz, R. M., and Ashbolt, N. J. (2014). Your garden hose: A potential health risk due to legionella spp. growth facilitated by free-living amoebae. Environ. Sci. Technol. 48, 10456-10464. doi: 10.1021/ es502652n

Tsao, H. F., Scheikl, U., Herbold, C., Indra, A., Walochnik, J., and Horn, M. (2019). The cooling tower water microbiota: seasonal dynamics and cooccurrence of bacterial and protist phylotypes. Water Res. 159, 464-479. doi: $10.1016 /$ j.watres.2019.04.028

United Nations (2021). Sustainable Development Goals Report 2021. 978-92-1-101439-6.

van der Kooij, D., Bakker, G. L., Italiaander, R., Veenendaal, H. R., and Wullings, B. A. (2017). Biofilm composition and threshold concentration for growth of legionella pneumophila on surfaces exposed to flowing warm tap water without disinfectant. Appl. Environ. Microbiol. 83. doi: 10.1128/AEM.02737-16

Vettrovský, T., and Baldrian, P. (2013). The variability of the $16 \mathrm{~S}$ rRNA gene in bacterial genomes and its consequences for bacterial community analyses. PLoS One 8:e57923. doi: 10.1371/journal.pone.0057923

Waak, M. B., Hozalski, R. M., Hallé, C., and Lapara, T. M. (2019). Comparison of the microbiomes of two drinking water distribution systems - With and without residual chloramine disinfection. Microbiome 7, 1-14. doi: 10.1186/ s40168-019-0707-5

Wagner, E. M., Fischel, K., Rammer, N., Beer, C., Lena, A., Conrady, B., et al. (2021). Bacteria of eleven different species isolated from biofilms in a meat processing environment have diverse biofilm forming abilities. Int. J. Food Microbiol. 349:109232. doi: 10.1016/j.ijfoodmicro.2021.109232

Wagner, E. M., Pracser, N., Thalguter, S., Fischel, K., Rammer, N., Pospíšilová, L., et al. (2020). Identification of biofilm hotspots in a meat processing environment: detection of spoilage bacteria in multi-species biofilms. Int. J. Food Microbiol. 328:108668. doi: 10.1016/j.ijfoodmicro. 2020.108668

Wickham, H. (2016). ggplot2: Elegant Graphics for Data Analysis. Springer-Verlag New York. Available at: https://ggplot2.tidyverse.org (Accessed January11, 2022).

Wingender, J., and Flemming, H. C. (2004). Contamination potential of drinking water distribution network biofilms. Water Sci. Technol. 49, 277-286. doi: $10.2166 /$ wst.2004.0861

Wingender, J., and Flemming, H. C. (2011). Biofilms in drinking water and their role as reservoir for pathogens. Int. J. Hyg. Environ. Health 214, 417-423. doi: 10.1016/j.ijheh.2011.05.009 
Zwirzitz, B., Wetzels, S. U., Dixon, E. D., Fleischmann, S., Selberherr, E., Thalguter, S., et al. (2021). Co-occurrence of listeria spp. and spoilage associated microbiota during meat processing due to cross-contamination events. Front. Microbiol. 12. doi: 10.3389/fmicb.2021.632935

Conflict of Interest: The authors declare that the research was conducted in the absence of any commercial or financial relationships that could be construed as a potential conflict of interest.

Publisher's Note: All claims expressed in this article are solely those of the authors and do not necessarily represent those of their affiliated organizations, or those of the publisher, the editors and the reviewers. Any product that may be evaluated in this article, or claim that may be made by its manufacturer, is not guaranteed or endorsed by the publisher.

Copyright (๑) 2022 Voglauer, Zwirzitz, Thalguter, Selberherr, Wagner and Rychli. This is an open-access article distributed under the terms of the Creative Commons Attribution License (CC BY). The use, distribution or reproduction in other forums is permitted, provided the original author(s) and the copyright owner(s) are credited and that the original publication in this journal is cited, in accordance with accepted academic practice. No use, distribution or reproduction is permitted which does not comply with these terms. 